\title{
Qous (2019)
}

\section{Anaïs Tillier}

\section{OpenEdition Journals}

\section{Édition électronique}

URL : https://journals.openedition.org/baefe/1079

DOI : 10.4000/baefe.1079

ISSN : 2732-687X

Éditeur

ResEFE

\section{Référence électronique}

Anaïs Tillier, « Qous (2019) » [notice archéologique], Bulletin archéologique des Écoles françaises à l'étranger [En ligne], Égypte, mis en ligne le 05 octobre 2020, consulté le 28 juillet 2021. URL : http:// journals.openedition.org/baefe/1079 ; DOI : https://doi.org/10.4000/baefe.1079

Ce document a été généré automatiquement le 28 juillet 2021.

\section{c) (†)}

Le Bulletin archéologique des Écoles françaises à l'étranger est mise à disposition selon les termes de la Licence Creative Commons Attribution - Pas d'Utilisation Commerciale - Pas de Modification 4.0 International. 


\title{
Qous (2019)
}

\author{
Anaïs Tillier
}

\section{NOTE DE L'AUTEUR}

Année de la campagne : 2019 (13 janvier - 24 janvier)

Numéro et intitulé de l'opération de terrain : 17323 - Étude de faisabilité de la publication des monuments antiques de Qous

Composition de l'équipe de terrain : L'équipe était composée d'Anaïs Tillier (membre associé UMR 5140 Archéologie des sociétés méditerranéennes, équipe Égypte nilotique et méditerranéenne, CNRS-Université Paul-Valéry Montpellier 3), Dr Ali Abdelhalim Ali (co-directeur de la mission, égyptologue, université Ayn Shams), Mohamed Abd elHamid Hamada (coordinateur local, ministère des Antiquités de l'Égypte).

Le ministère des Antiquités était représenté par Ahmed Said, accompagné de deux stagiaires Eman Saber Mohamed et Fatma Tony Ahmed. Ahmed Khalifa, restaurateur du département des projets de Qift-Qena, est intervenu pendant les travaux. Ayman Hendi, chef du bureau de Qena, et Ashraf Nasr de l'inspectorat du ministère des Antiquités à Qena ont apporté leur aide et soutien au déroulement de la mission. Le raïs Omar Farouk de Karnak a conduit les travaux.

Partenariat institutionnel : La mission bénéficie du soutien de l'université de Ayn Shams (Le Caire).

Sponsor : Fonds Khéops pour l'archéologie (Paris)

Etablissements porteurs du projet : Université d'Ayn Shams (Le Caire) / IFAO

1 Le projet initialement prévu en mai 2017 a été annulé et reporté en raison du retard des autorisations. La mission de terrain s'est déroulée du 13 au 24 janvier 2019 grâce au soutien de l'Ifao et du fonds Khéops pour l'archéologie. Les travaux se sont concentrés sur le secteur de la porte est, le plus accessible et actuellement protégé par une enceinte, avec pour principal objectif le déplacement et la protection des blocs gisant au sol et affectés par les remontées d'eau et de sels (fig. 1). 
Fig. 1. Localisation des principaux monuments de Qous (A. Tillier).

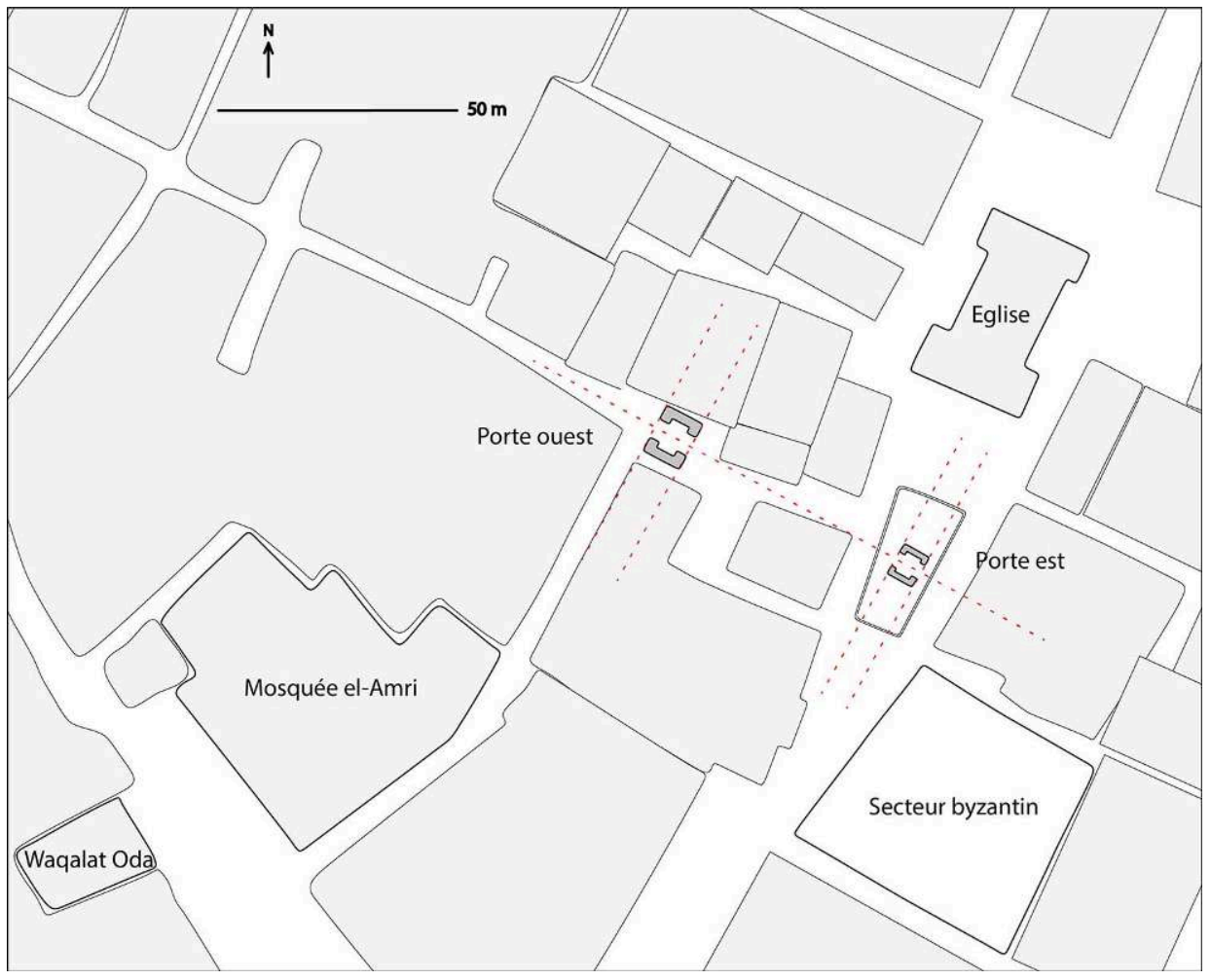

(C) Ifao. 17323_2019_NDMCN_001

\section{Secteur de la porte est}

(fig. 2)

L'inspecteur Ahmed Said indique que ce secteur recouvre une zone plus étendue que celle délimitée par l'enceinte actuelle, allant jusqu'à l'église située au nord. La construction d'un nouveau mur d'enceinte correspondant à cette zone a été estimée à 5000 euros.

3 La surface du site a été nettoyée des déchets dus à la proximité des habitations modernes. Environ une quarantaine de blocs de pierre de taille variable se trouvaient au sol, exposés aux remontées de sels. Les éléments de taille moyenne $(20-60 \mathrm{~cm})$ ont été installés sur la banquette construite par le ministère des Antiquités (MoA) quelques années auparavant. Une douzaine de blocs portent les restes de décor et d'inscriptions hiéroglyphiques. L'un d'entre eux complète la scène du second registre du montant nord-ouest.

La mission a fait réaliser deux banquettes supplémentaires pour accueillir cinq fragments imposants de la corniche à gorge de la porte (env. $150 \times 100 \times 70 \mathrm{~cm}$ ). Deux d'entre eux ont été déplacés; un troisième sur le point de se déliter a reçu un traitement de consolidation; un quatrième, plus massif, n'a pu être déplacé avec le matériel disponible durant la mission. Son dégagement partiel a permis d'observer le début d'une inscription grecque, sans doute celle publiée par les savants de la Description de l'Égypte et par Champollion, et disparue ensuite, confirmant ainsi l'identité de l'unique porte recensée à Qous par les voyageurs des $\mathrm{XVIII}^{\mathrm{e}}$ et $\mathrm{XIX}^{\mathrm{e}}$ siècles. 
Fig. 2. Porte est, vue nord-sud (2019) (A. Tillier).

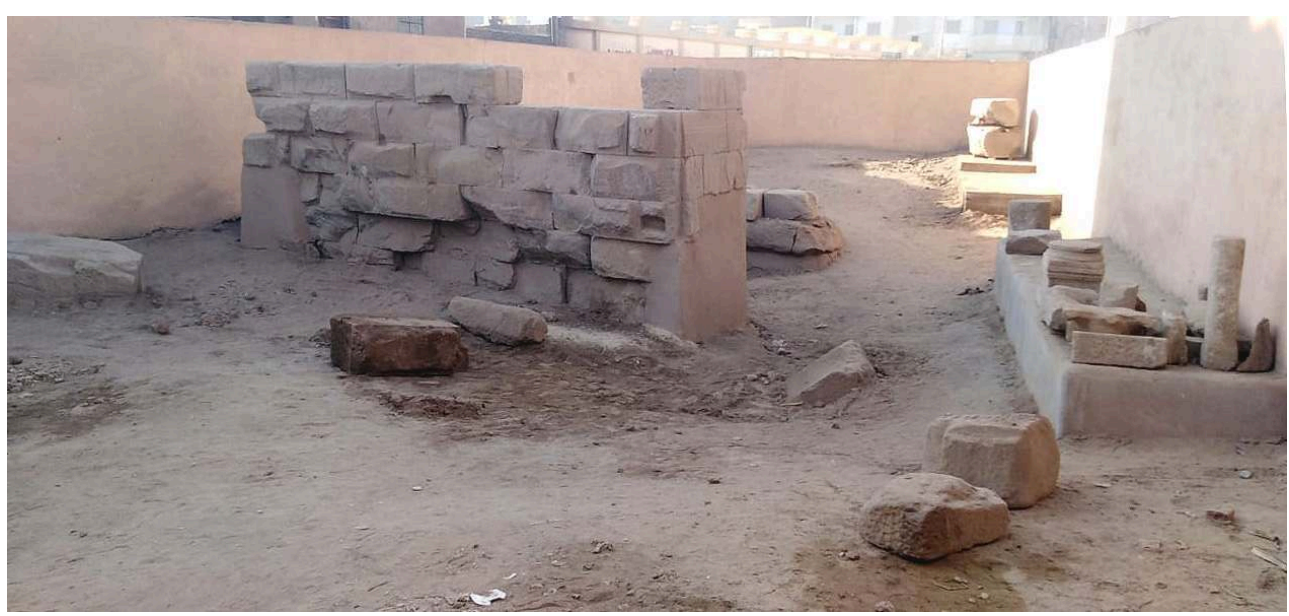

C Ifao. 17323_2019_NDMSE_001

5 Le déplacement du groupe de blocs situés dans l'angle sud-est a conduit à la découverte d'un vase en pierre, affleurant la surface et contenant trois fioles en verre d'époque islamique (fig. 3).

Fig. 3. Vase en pierre et fioles islamiques trouvés près de l'angle sud-est de la porte est (A. Tillier).

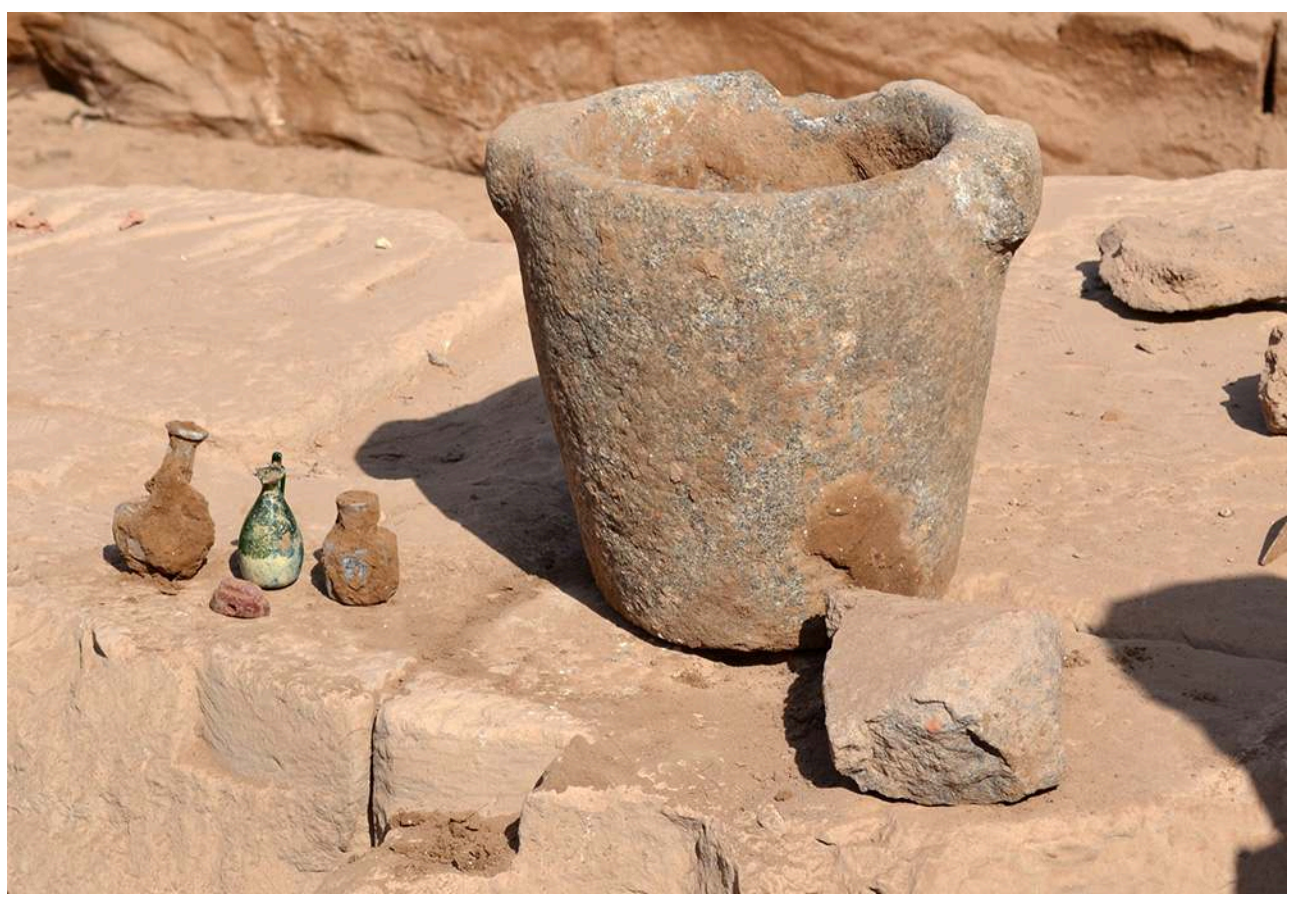

(C) Ifao. 17323_2019_NDMDM_001

\section{Formation, coopération}

6 La réalisation d'un premier inventaire des blocs a permis de former l'inspecteur Ahmed Said et ses deux stagiaires aux méthodes de documentation, ainsi qu'à la lecture du décor et des textes hiéroglyphiques des temples ptolémaïques. 
7 Nous ont été rapportées également des informations sur les fouilles clandestines régulièrement enregistrées par l'inspectorat. L'une d'entre elles, signalée en 2014 et localisée à $5 \mathrm{~m}$ de la porte ouest, avait mis au jour trois blocs inscrits d'environ $1 \mathrm{~m}$ en parfait état. Une vidéo de la découverte a permis d'identifier deux blocs comme des assises de la porte et le troisième, gravé en relief levé, appartient à l'intérieur d'une chapelle ou d'une pièce du temple encore inconnue (fig. 4). Les blocs sont restés en place sous une habitation délabrée du début du xx siècle qui a été démolie en 2018 par son propriétaire avec l'intention d'en construire une nouvelle en briques et en béton. Le projet immobilier est aujourd'hui à l'arrêt en raison du rapport du MoA.

Fig. 4. Porte ouest, vue du sud-ouest, avec au fond l'emprunte visible de la maison démolie sous laquelle reposent plusieurs blocs (2019) (A. Tillier).

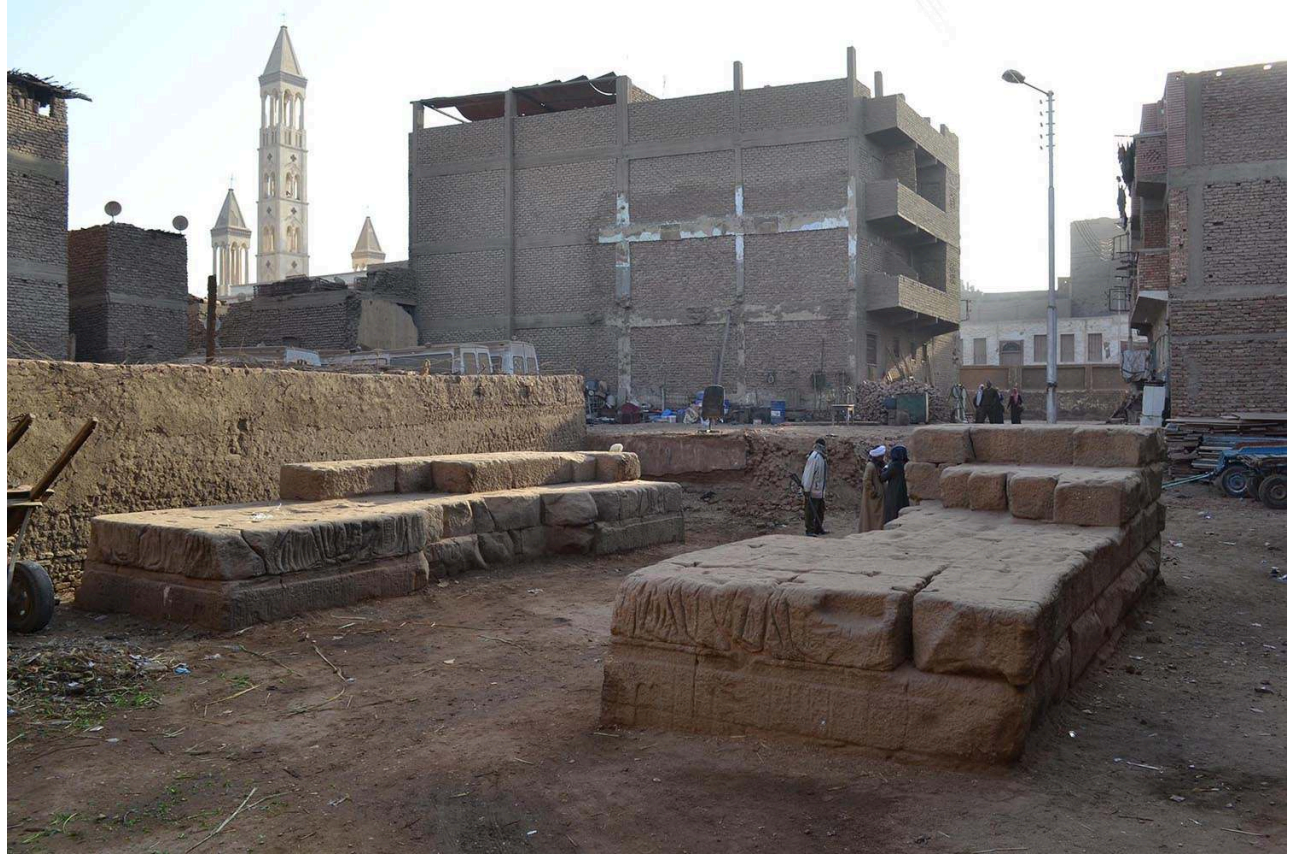

(C) Ifao. 17323_2019_NDMSE_002

\section{Publications}

Anaïs Tillier, « Qous », in Laurent Coulon, Mélanie Cressent (éd.), Archéologie française en Égypte, BiGen 59, Le Caire, Ifao, 2019, p. 176-181.

\section{Diffusion}

Carnet de recherche : http://qus.hypotheses.org 


\section{BIBLIOGRAPHIE}

Anaïs Tillier, « Enquête sur le nom et les graphies de l'ancienne Gsy (Qous) », BIFAO 113, 2013, p. 433-447.

Anaïs Tillier, « Haroéris de Qous et la XIX province de Haute Égypte », in Núria Castellano, Maite Mascort, Concepció Piedrafita, Jaume Vivó (éd.), Ex Aegypto lux et sapientia. Homenatge al professor Josep Padró Parcerisa, Nova Studia Aegyptiaca IX, Barcelone, Universitat de Barcelona, 2015, p. 553-557.

\section{INDEX}

Année de l'opération : 2019

anthroponymes https://ark.frantiq.fr/ark:/26678/pcrtLiJJ1wLfwi chronologie https://ark.frantiq.fr/ark:/26678/pcrtOD0uWt4YFl sujets https://ark.frantiq.fr/ark:/26678/crtqPFI1HPTo2, https://ark.frantiq.fr/ark:/26678/ pcrtwXskswbuF3, https://ark.frantiq.fr/ark:/26678/pcrtYhYMiLwDUr, https://ark.frantiq.fr/ ark:/26678/pcrt4otVkR3bqD, https://ark.frantiq.fr/ark:/26678/pcrtKoEikIOiIm, https:// ark.frantiq.fr/ark:/26678/crtc5yIIsRRgg, https://ark.frantiq.fr/ark:/26678/pcrt4GqimUgoOq

Thèmes : IFAO

\section{AUTEURS}

\section{ANAIIS TILLIER}

Membre associé UMR 5140 Archéologie des sociétés méditerranéennes, équipe Égypte nilotique et méditerranéenne, CNRS-Université Paul-Valéry Montpellier 3 